\title{
Automated Scanning and Individual Identification System for Parts without Marking or Tagging
}

\author{
Kengo Makino \\ NEC Corporation \\ Kawasaki, Japan \\ k-makino@mb.jp.nec.com \\ Toru Takahashi \\ NEC Corporation \\ Kawasaki, Japan \\ t-takahashi@hg.nec.com
}

\author{
Wenjie Duan \\ Delft University of Technology \\ Delft, Netherlands \\ W.Duan-1@student.tudelft.nl
Yuta Kudo
NEC Corporation
Kawasaki, Japan
y-kudo@bc.jp.nec.com

\author{
Rui Ishiyama \\ NEC Corporation \\ Kawasaki, Japan \\ r-ishiyama@bl.jp.nec.com \\ Pieter Jonker \\ Delft University of Technology \\ Delft, Netherlands \\ p.p.jonker@tudelft.nl
}

\begin{abstract}
This paper presents a fully automated system for detecting, classifying, microscopic imaging, and individually identifying multiple parts without ID-marking or tagging. The system is beneficial for product assemblers, who handle multiple types of parts simultaneously. They can ensure traceability quite easily by only placing the parts freely on the system platform. The system captures microscopic images of parts as their "fingerprints," which are matched with pre-registered images in a database to identify an individual part's information such as its serial number. We demonstrate a working prototype and interaction scenario.
\end{abstract}

\section{CCS CONCEPTS}

- Computing methodologies $\rightarrow$ Object identification;

\section{KEYWORDS}

IoT; Traceability; Image recognition; Individual identification; Classification; Objects localization; Fingerprint of Things

\section{ACM Reference Format:}

Kengo Makino, Wenjie Duan, Rui Ishiyama, Toru Takahashi, Yuta Kudo, and Pieter Jonker. 2018. Automated Scanning and Individual Identification System for Parts without Marking or Tagging. In ICMR '18: 2018 International Conference on Multimedia Retrieval, fune 11-14, 2018, Yokohama, Japan. ACM, New York, NY, USA, 4 pages. https://doi.org/10.1145/3206025.3206088

\section{INTRODUCTION}

Traceability of individual parts is essential for ensuring quality and safety of assembled products. For example, to determine the cause of a product failure, information about the individual parts used in the assembly process must be retrieved. The conventional method for ensuring parts traceability is attaching ID tags such as barcodes, radio frequency ID (RFID) tags, etc. onto the parts and

Permission to make digital or hard copies of all or part of this work for personal or classroom use is granted without fee provided that copies are not made or distributed for profit or commercial advantage and that copies bear this notice and the full citation on the first page. Copyrights for components of this work owned by others than ACM must be honored. Abstracting with credit is permitted. To copy otherwise, or republish, to post on servers or to redistribute to lists, requires prior specific permission and/or a fee. Request permissions from permissions@acm.org.

ICMR '18, fune 11-14, 2018, Yokohama, Japan

(C) 2018 Association for Computing Machinery.

ACM ISBN 978-1-4503-5046-4/18/06 . .\$15.00

https://doi.org/10.1145/3206025.3206088

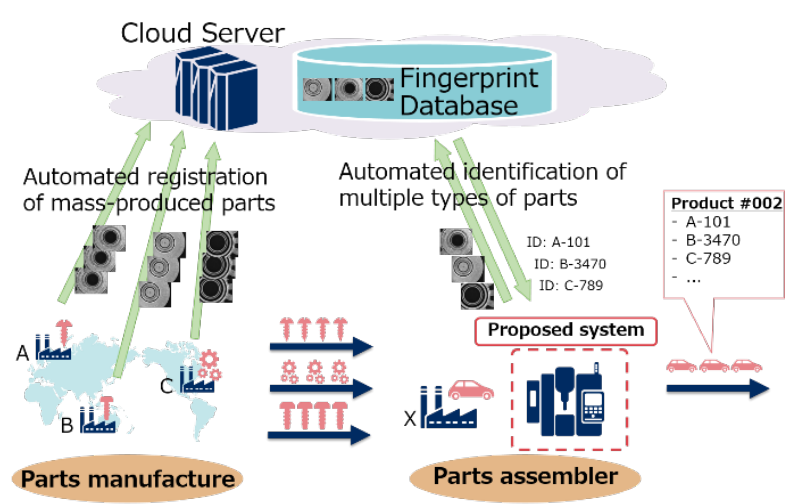

Figure 1: Concept of parts traceability throughout supply chain using the proposed system.

reading them. Marking or tagging makes parts identification accurate and convenient. However, these approach cannot be applied to mass-production use, especially in case of small parts. Since characters, barcodes or tags have to be further smaller as parts become smaller, marking and reading them becomes increasingly difficult thus costly. Therefore, total cost for marking or reading will become prohibitive as the amount of production increase. A fully automated identification system without marking or tagging is demanded for mass-production use.

Some emerging identification techniques have been proposed to enable tag-less identification of objects $[1,3,8,12]$. These techniques use unique surface patterns naturally formed during the manufacturing process. Bunchanan et al. [3] proposed a document and package identification technique that uses laser speckle patterns. Beekhof et al. [1] proposed a basic framework to identify papers using random microstructures of fibers. Matsumoto et al. [8] proposed a nano-artifact metrics based on the random collapse of resist pillars. Furthermore, a fingerprint-based identification method has been proposed for metal parts that are widely used in industrial products [12]. This method has been applied to ensuring traceability and preventing misuse of parts [11].

Automation is mandatory for use in mass-production. If an operator of a factory line manually places parts under a camera to capture a fingerprint image, the operator must precisely adjust 


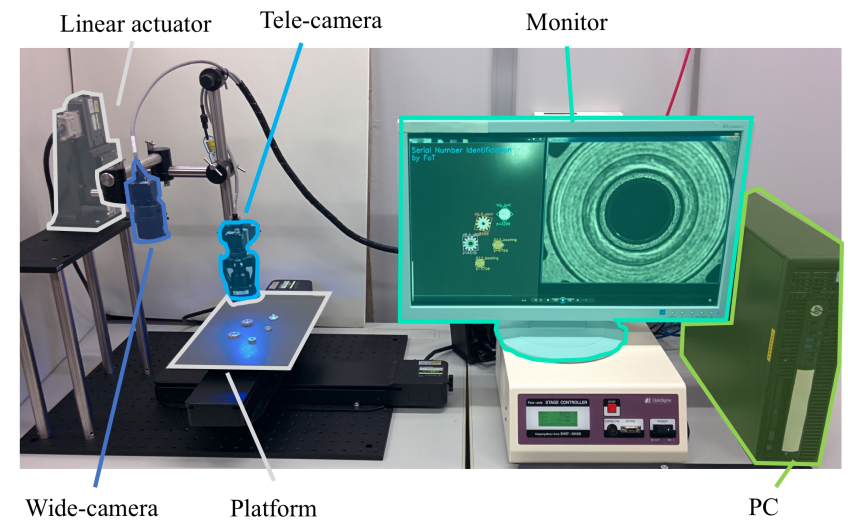

Figure 2: Hardware components of the system.

the part's position and the camera's focus (we call this process alignment). This is because the microscopic camera's field of view and depth of field are very narrow. Such manual alignment is time consuming and detrimental to assembly efficiency. To use the fingerprint identification in a real assembly process, a fully automated capturing system is needed to solve this problem.

Of the aforementioned tag-less identification methods, only the fingerprint-based identification method [12] has been fully automated. The prototype machine [12] can capture fingerprint images for numerous bolts with automated feeding. It is designed for parts manufacturers to register fingerprints and parts information, as it can handle only a single type of mass-produced parts. It is too limited for assembling, which requires handling multiple part types simultaneously. Developing dedicated hardware for every type of parts is too costly.

This paper proposes a fully automated identification system that can scan and identify multiple types of parts with a single general setup. The proposed system first automatically detects part position and type by using a wide-view camera and adjusts the position of the microscopic camera to capture a fingerprint image. Using the fingerprint image, the proposed system also automatically identifies individuals of those parts. As the operator needs only to freely place parts on the platform of our system, the system ensures traceability without any costly manual operation.

Figure 1 shows the concept of the parts traceability system using our parts identification system. First, fingerprint images of massproduced parts are registered in the database during the parts' manufacturing processes. When the parts are picked out during the assembly process, fingerprint images of the parts are captured and identified automatically by our proposed system. This enables the parts assembler to identify all constituent parts of any product, thus the traceability is ensured throughout the supply chain.

\section{SYSTEM}

Our proposed system consists of a platform, a linear actuator, a desktop PC with a display monitor, and two cameras. Figure 2 shows the hardware components of the system. Figure 3 shows the workflow of the system. First, the operator freely places parts

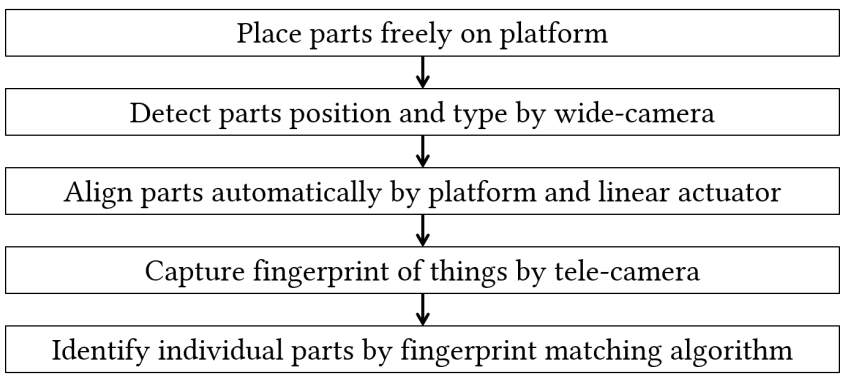

Figure 3: Overview of the proposed system.

on the platform. Then, a wide-view camera (referred to as a widecamera from here on) detects and classifies the positions and types of the parts. This process will be further described in section 2.1. The other camera equipped with a microscopic lens (referred to as a tele-camera from here on) captures a microscopic image of a part's surface. The platform and tele-camera move simultaneously to align the part and bring it into focus, the details of which will be described in section 2.2. Captured microscopic images are individually unique and considered as parts' "fingerprints," which we will call "fingerprint images" from here on. These fingerprint images will be matched with pre-registered images in a database. The matching process will be further described in section 2.3. The PC processes images from the cameras and controls the movements of the linear actuator and platform. The monitor shows the results of part detection, captured fingerprint images, and individual part IDs to the operator in real-time.

\subsection{Detection of Part Position and Type by Wide-camera}

The wide-camera monitors the whole platform to detect where parts are placed and classifies part types in real-time. The detected positions and classified types will be used to align the parts with the tele-camera and to capture a fingerprint image.

We use background subtraction, binarization, and morphological operations to extract part regions in a captured image. Then, a contour detection method [10] is used to detect the center position and region of each part. The center position is used to align parts. Lens distortion of the wide-camera is corrected by the camera calibration method [13], so the detected part's position is accurate enough for aligning the part with the tele-camera's view, described in section 2.2 .

The detected regions of parts in the image are cropped out before part type is classified. We use the bag of features method [7] and the support vector machine classifier [5]. In the bag of features method, we use the Oriented FAST and Rotated BRIEF (ORB) feature [9] as our feature point detector and descripter.

In our demonstration, the detection and classification results are displayed on the monitor in real-time. Figure 7 (a) shows the interface of the detection mode. The part types are shown in different colors. 


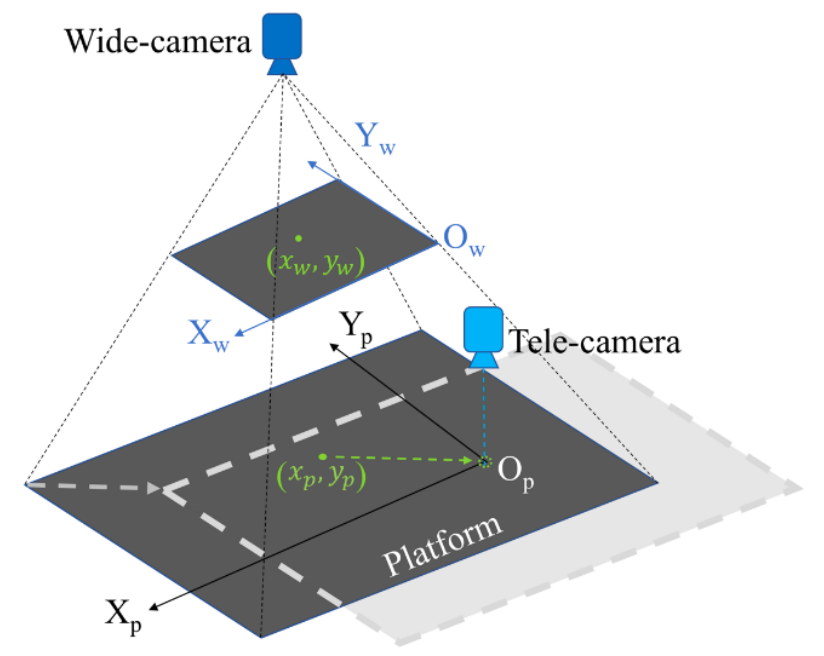

Figure 4: Graph for wide-camera coordinate and platform coordinate. Green point is on platform and is detected by wide-camera at $\left(x_{w}, y_{w}\right)$. Its relative position to tele-camera is $\left(x_{p}, y_{p}\right)$. After platform moves to dashed position, green point is aligned with tele-camera.

\subsection{Automatic Microscopic Fingerprint Capturing by Tele-camera}

After detecting all part positions and types, the system captures fingerprint images. First, the platform automatically moves in the horizontal plane to align parts with the focal position of the telecamera. This alignment needs to be automated, because the field of view of the tele-camera is very small (about $10 \mathrm{~mm} \times 10 \mathrm{~mm}$ ). Simultaneously, the tele-camera's height is adjusted by a linear actuator to align the tele-camera's focal distance with the part's top surface.

Let us define two coordinate systems: wide-camera coordinate and platform coordinate, as shown in Figure 4. The wide-camera coordinate is the wide-camera's image coordinate. The platform coordinate indicates a part's relative position to $\mathrm{O}_{\mathrm{p}}$, which is the focal position of the tele-camera on the platform. The origin of the platform coordinate is $\mathrm{O}_{\mathrm{p}}$. Using the methods described in section 2.1, we can obtain the part position in wide-camera coordinate $\left(x_{w}, y_{w}\right)$. To align the part with the tele-camera, we need the part position in platform coordinate $\left(x_{p}, y_{p}\right)$. We use a preset $3 \times 3$ homography matrix $\boldsymbol{H}$ to map a point from $\left(x_{w}, y_{w}\right)$ to $\left(x_{p}, y_{p}\right)$ as follows.

$$
\left[\begin{array}{l}
x_{p} \\
y_{p} \\
1
\end{array}\right]=\boldsymbol{H}\left[\begin{array}{l}
x_{w} \\
y_{w} \\
1
\end{array}\right],
$$

$\boldsymbol{H}$ is calibrated beforehand by using eight points marked on the platform. The eight points' positions in wide-camera coordinates $\left(x_{w}, y_{w}\right)$ are obtained by detecting the marks in a captured image. Next, we manually control the platform to align the marks with the tele-camera's focal position, where the part is captured at image center. We then read the traveled distances of the platform from the platform's encoder. The distances give us the mark's position

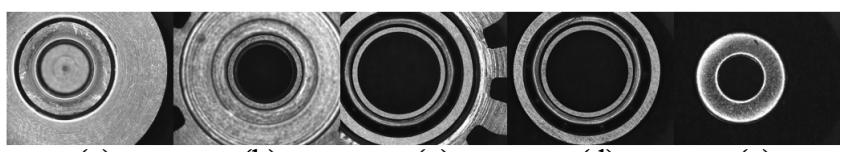

(a)

(b)

(c)

(d)

(e)

Figure 5: Examples of tested parts' fingerprint images. (a) big bolt; (b) d4.4 gear; (c) d6.4 gear; (d) D13 bearing; (e) washer.

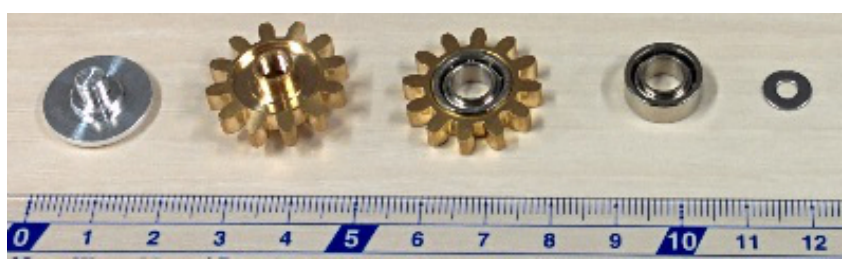

Figure 6: Tested parts types. From left to right are big bolt, d4.4 gear, d6.4 gear, D13 bearing, and washer.

in platform coordinates $\left(x_{p}, y_{p}\right)$. After obtaining 16 coordinates of the 8 marks, we estimate the homography $\boldsymbol{H}$ by minimizing the back-projection error of Equation (1) by using the least squares method [2] and preset the values of $\boldsymbol{H}$ in the system.

Using the homography $\boldsymbol{H}$, we can calculate a part's relative position to the tele-camera and control the platform to align the part with the focal position of the tele-camera.

The linear actuator adjusts the tele-camera's height in accordance with part type, because the lens of the tele-camera is fixedfocus and its depth of field is very narrow $(<1 \mathrm{~mm})$. To capture in-focus fingerprint images for multiple types of parts having different heights, the tele-camera's height needs to be adjusted. The appropriate heights of the tele-camera for all types of the parts are preset. Once a part is in focus, a fingerprint image will be captured as shown in Figure 5.

\subsection{Fingerprint Matching}

A captured fingerprint image is matched with each of the preregistered images in the database, and the individual identifier of the best matched part is obtained. The identifier specifies the individual part's information such as a serial number, which is displayed on the monitor.

For matching fingerprint images, we use a phase only correlation based image matching algorithm. As the parts are freely placed on the platform, the matching algorithm must be robust to rotations. To achieve such robustness, we use the method using Fourier-Mellin transform to estimate scale and rotation between the matched images $[4,6]$. We use the peak correlation value obtained in the estimation as the matching score. The matching scores are calculated between a captured image and all database images, thus the best match is determined in accordance with the highest score. The identifier specifies the individual part's information, which is displayed on the monitor.

\section{EXPERIMENTS}

We tested our proposed system with 170 individual parts of five different types of mechanical parts. We used 20 big bolts, $10 \mathrm{~d} 4.4$ 


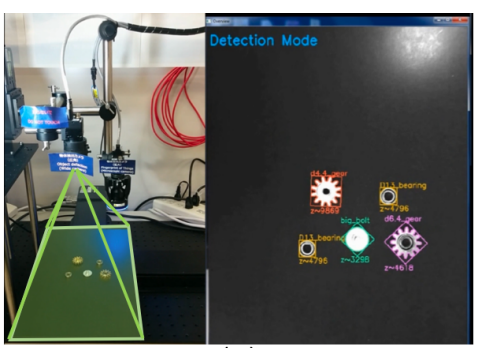

(a)

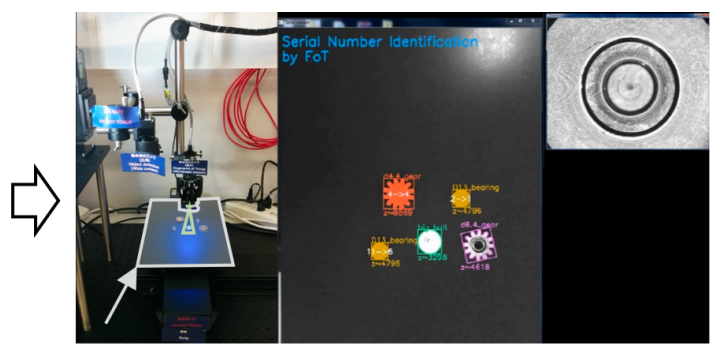

(b)

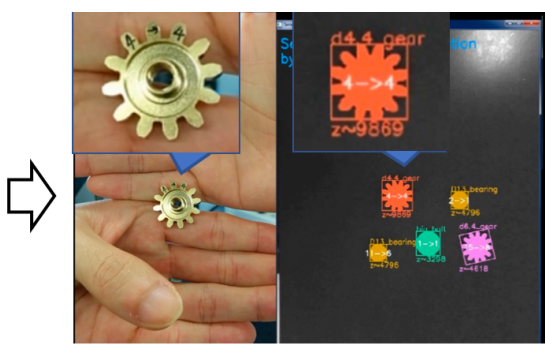

(c)

Figure 7: Demonstration of automated individual identification system. (a) Parts are placed freely on platform. (b) Platform moves parts to focal position of tele-camera and capture fingerprint image. (c) All parts are identified, and identification results are checked against ground truth.

gears, $20 \mathrm{~d} 6.4$ gears, 20 D13 bearing, and 100 washers. The washer is a steel flat washer that is commonly sold in hardware stores. The other four types of parts are taken from 10 disassembled commonly sold spinner toys. Figure 6 shows how these parts look.

20 images were captured for each of 5 parts types by wide-camera, thus 100 training images in total were used for training the classifier. The 20 images come from the one individual that was randomly chosen. The individual was randomly placed on the platform for 20 times to be captured with different appearances to the wide-camera due to changes of the viewing angle.

In the experiments, 170 individual parts were randomly placed on the platform, and all the parts were correctly detected, classified, and identified. The automated position detection proved sufficiently accurate, and all the five types were classified without error. This enabled us to capture fingerprint images without troublesome misalignment and miss-focus, and all the parts were successfully identified.

\section{DEMONSTRATION}

At the start of a demonstration, an operator can randomly choose some parts from all the pre-registered parts, which are 170 individuals of five different part types, and freely place them on the platform. The part detection and type classification are processed in real-time, and results are shown on the monitor, as shown in the right half of Figure 7 (a).

On the basis of the detection and classification results, the platform starts to align the parts with the tele-camera's focal position. The tele-camera's height is simultaneously adjusted with the part's surface height, which differs depending on the part type. After each part is aligned with the focal position of the tele-camera, a fingerprint image is captured. A captured fingerprint image is shown at the right side of the interface as shown in the right of Figure 7 (b). Matching runs in a background process, and the identification results are displayed on the monitor immediately. As shown in the center of Figure 7 (b), identified parts are colored and tagged with their individual information. For example, "2->1" indicates the part's own serial number is No. 2 and it is disassembled from assembly spinner No. 1 .

After the all chosen parts are identified, the operator can check the identification results against the ground truth of the serial number written on the back side of each part. As shown in Figure
7 (c), the tag of d4.4 gear is "4->4," which is identical to the serial number written on the part.

\section{CONCLUSION}

We have proposed a fully automated parts identification system that requires neither tagging nor additional operation costs for identification. An operator only needs to freely place parts on the platform, then the system automatically identifies the serial numbers of those parts. Since the system can handle multiple types of parts simultaneously, it helps product assemblers ensure total traceability throughout the supply chain.

The system currently can only capture the parts' top surface that is paralleled to the platform. Detection of orientation of parts' top surface and 6-d alignment with the tele-camera remains as our future works.

\section{REFERENCES}

[1] F. Beekhof et al. 2008. Secure surface identification codes. In Security, Forensics, Steganography, and Watermarking of Multimedia Contents X, Vol. 6819. International Society for Optics and Photonics, 68190D.

[2] G. Bradski et al. 2008. Learning OpenCV: Computer vision with the OpenCV library. " O’Reilly Media, Inc.".

[3] J.D.R. Buchanan et al. 2005. Forgery:'fingerprinting' documents and packaging. Nature 436, 7050 (2005), 475.

[4] D. Casasent et al. 1977. New optical transforms for pattern recognition. Proc. of the IEEE 65, 1 (Jan. 1977), 77-84.

[5] N. Cristianini et al. 2000. An introduction to support vector machines and other kernel-based learning methods. Cambridge university press.

[6] H.T. Ho et al. 2008. Optical flow estimation using Fourier Mellin transform. In Proc. of the 2008 IEEE Conf. on Computer Vision and Pattern Recognition (CVPR). IEEE, 1-8.

[7] F-F. Li et al. 2005. A bayesian hierarchical model for learning natural scene categories. In Proc. of 2005 IEEE Computer Society Conf. on Computer Vision and Pattern Recognition (CVPR), Vol. 2. IEEE, 524-531.

[8] T. Matsumoto et al. 2014. Nano-artifact metrics based on random collapse of resist. Scientific reports 4 (Aug. 2014), 6142.

[9] E. Rublee et al. 2011. ORB: An efficient alternative to SIFT or SURF. In Proc. of 2011 Int. Conf. on Computer Vision (ICCV). IEEE, 2564-2571.

[10] S. Suzuki et al. 1985. Topological structural analysis of digitized binary images by border following. Computer vision, graphics, and image processing 30, 1 (Apr. 1985), 32-46.

[11] T. Takahashi et al. 2016. Intelli-Wrench: Smart Navigation Tool for Mechanical Assembly and Maintenance. In Proc. of the 24th Annual ACM Int. Conf. on Multimedia (ACMMM). ACM, 752-753.

[12] T. Takahashi et al. 2017. Mass-produced parts traceability system based on automated scanning of "Fingerprint of Things". In Proc. of Fifteenth IAPR International Conference on Machine Vision Applications (MVA). IEEE, 202-206.

[13] Z. Zhang. 2000. A flexible new technique for camera calibration. IEEE Trans. on pattern analysis and machine intelligence (PAMI) 22, 11 (Nov. 2000), 1330-1334. 